\title{
On Katugampola Laplace transform
}

\author{
Mohammed S. El-Khatib ${ }^{a, *}$, Tariq O. Salim ${ }^{b}$, Atta A.K. Abu Hany ${ }^{\mathrm{c}}$ \\ ${ }^{a}$ Department of Mathematics, Al-Azhar Universiry-Gaza, Palestine \\ ${ }^{b}$ Department of Mathematics, Al-Azhar Universiry-Gaza, Palestine \\ ${ }^{c}$ Department of Mathematics, Al-Azhar Universiry-Gaza, Palestine
}

\begin{abstract}
The aim of this article is to introduce a new form for the Laplace transform. This new definition will be considered as one of the generalizations of the usual (classical) Laplace transform. We employ the new "Katugampola derivative", which obeys classical properties and define Katugampola Laplace transform. We obtain some properties of this transform and find the relation between the Katugampola Laplace transform and the usual Laplace one.
\end{abstract}

Keywords: Katugampola derivative; Katugampola Laplace transform.

2010 MSC: 26A33, , 42A38,

\section{Introduction}

The derivative of non-integral order "Fractional derivative" is an interesting research topic since it is a generalization of the classical integer calculus. Several types of fractional derivatives were introduced and studied by Riemann-Liouville, Caputo, Hadamard, Weyl, and Grünwald-Letnikov; for more details one can see $[4,6,7,8]$. Unfortunately all these fractional derivatives fail to satisfy some basic properties of the classical integer calculus like product rule, quotient rule, chain rule, Roll's theorem, mean-value theorem and composition of two functions. Also, those fractional derivatives inherit non-locality and most of them propose that the derivative of a constant is not zero. Those inconsistencies lead to some difficulties in the applications of fractional derivatives in physics, engineering and real world problems.

To overcome all the difficulties raised, Khalil et al. [5] introduced and investigated the so called conformable fractional derivative and also, Katugampola [3] introduced and studied a similar type of derivative, later called Katugampola derivative and is defined as follows

Definition 1.1 [3] Let $f:[0, \infty) \rightarrow R$ and $t>0$. Then, the Katugampola derivative of $f$ of order $\alpha$ is defined by

$$
D^{\alpha} f(t)=\lim _{\varepsilon \rightarrow 0} \frac{f\left(t e^{\varepsilon t^{-\alpha}}\right)-f(t)}{\varepsilon}
$$

\footnotetext{
*Corresponding author

Email addresses: msmkhatib@yahoo.com (Mohammed S. El-Khatib), trsalim@yahoo.com (Tariq O. Salim), attahany@gmail. com (Atta A.K. Abu Hany)
}

doi:10.31559/glm2020.9.2.5 
for $t>0$ and $\alpha \in(0,1]$. If $f$ is $\alpha$-differentiable in some $(0, a), a>0$ and $\lim _{t \rightarrow 0^{+}} D^{\alpha}(f)(t)$ exists, then $D^{\alpha}(f)(0)=\lim _{t \rightarrow 0^{+}} D^{\alpha}(f)(t)$.

Definition 1.2[3] Let $\alpha \in(n, n+1$ ], for some $n \in N$ and $f$ be an $n$-differentiable at $t>0$. Then the $\alpha$-fractional derivative of $f$ is defined by

$$
D^{\alpha} f(t)=\lim _{\varepsilon \rightarrow 0} \frac{f^{(n)}\left(t e^{\varepsilon t^{n-\alpha}}\right)-f^{(n)}(t)}{\varepsilon}
$$

if the limit exists.

Note that Katugampola derivative satisfies product rule, quotient rule, chain rule,... etc. and it is consistent in its properties with the classical calculus of integer order. In addition, we have the following theorem.

Theorem 1.3 Let $\alpha \in(n, n+1]$, for some $n \in N$ and $f$ be an $(n+1)$-differentiable at $t>0$. Then,

$$
D^{\alpha} f(t)=t^{n+1-\alpha} f^{(n+1)}(t)
$$

Proof.

$$
\begin{aligned}
D^{\alpha} f(t) & =\lim _{\varepsilon \rightarrow 0} \frac{f^{(n)}\left(t \cdot e^{\varepsilon \cdot t^{n-\alpha}}\right)-f^{(n)}(t)}{\varepsilon} \\
& =\lim _{\varepsilon \rightarrow 0} \frac{f^{(n)}\left(t+\varepsilon t^{n-\alpha+1}+\frac{\varepsilon^{2} t^{2 n-2 \alpha+1}}{2 !}+\frac{\varepsilon^{3} t^{3 n-3 \alpha+1}}{3 !}+\ldots\right)-f^{(n)}(t)}{\varepsilon} .
\end{aligned}
$$

Let $h=\varepsilon \mathrm{t}^{\mathrm{n}-\alpha+1}\left[1+\frac{\varepsilon \mathrm{t}^{\mathrm{n}-\alpha}}{2 !}+\frac{\varepsilon^{2} \mathrm{t}^{2 n-2 \alpha}}{3 !}+\ldots\right]$, so $\mathrm{h}=\varepsilon \mathrm{t}^{\mathrm{n}-\alpha+1}[1+\mathrm{O}(\varepsilon)]$,

where $h \rightarrow 0$ as $\varepsilon \rightarrow 0$. Hence,

$$
D^{\alpha} f(t)=t^{n-\alpha+1} \lim _{h \rightarrow 0} \frac{f^{(n)}(t+h)-f^{(n)}(t)}{h}=t^{n-\alpha+1} f^{(n+1)}(t) .
$$

\section{Katugampola Laplace Transform}

Salim, T.O., et al [9] have introduced a new definition of Katugampola Fourier transform which finds very interesting reputation between mathematicians. Following the same procedure, they continue their work and define a new Laplace transform called Katugampola Laplace transform. Some basic properties of this transform are given here. Abdeljawad [1] gave the definition of conformable Laplace transform.

In this section, we introduce and study the relation between Katugampola Laplace transform and the usual Laplace transform. The Katugampola Laplace transform of some functions are established and then we obtain a convolution formula for this transform. Remember here the usual Laplace transform to the function $f$,

$$
\operatorname{Lf}(x)\}(p)=\ell\{f(x)\}(p)=\int_{0}^{\infty} f(x) e^{-p x} d x .
$$

Definition 2.1 Let $\alpha \in(n, n+1]$ for some $n \in N$ and $f(t)$ be a real valued function on $[0, \infty)$. The Katugampola Laplace transform of $f(t)$ of order $\alpha$ is defined as

$$
\mathrm{L}_{\alpha}\{\mathrm{f}(\mathrm{t})\}(\mathrm{p})=\tilde{\mathrm{f}}(\mathrm{p})=\int_{0}^{\infty} e^{-p \frac{\mathrm{t}^{\alpha-n}}{\alpha-n}} f(\mathrm{t}) \mathrm{t}^{\alpha-n-1} d \mathrm{t} .
$$


Theorem 2.2 Let $\alpha \in(n, n+1]$, for some $n \in N$ and $f(x)$ be a real valued function on $[0, \infty)$. Then,

$$
\mathrm{L}_{\alpha}\left\{D^{\alpha} f(t)\right\}(p)=p L_{\alpha}\left\{f^{(n)}(t)\right\}(p)-f^{(n)}(0) .
$$

Proof. By using Definition 2.1 and Theorem 1.3, we have

$$
\begin{gathered}
L_{\alpha}\left\{D^{\alpha} f(t)\right\}(p)=L_{\alpha}\left\{t^{n-\alpha+1} f^{(n+1)}(t)\right\}(p) \\
=\int_{0}^{\infty} e^{-p \frac{t^{\alpha-n}}{\alpha-n}} t^{n-\alpha+1} f^{(n+1)}(t) t^{\alpha-n-1} d t \\
=\int_{0}^{\infty} e^{-p \frac{t^{\alpha-n}}{\alpha-n}} f^{(n+1)}(t) d t .
\end{gathered}
$$

Now by using integration by parts, we get

$$
\begin{gathered}
\mathrm{L}_{\alpha}\left\{D^{\alpha} f(t)\right\}(p)=\left.e^{-p \frac{t^{\alpha-n}}{\alpha-n}} f^{(n)}(t)\right|_{0} ^{\infty}+p \int_{0}^{\infty} e^{-p \frac{t^{\alpha-n}}{\alpha-n}} f^{(n)}(t) t^{\alpha-n-1} d t \\
=p L_{\alpha}\left\{f^{(n)}(t)\right\}(p)-f^{(n)}(0) .
\end{gathered}
$$

Corollary 2.3 Let $\alpha \in(0,1]$, and $f:[0, \infty] \rightarrow R$ be $\alpha$-differentiable real valued function. Then

$$
\mathrm{L}_{\alpha}\left\{\mathrm{D}^{\alpha}(\mathrm{f})(\mathrm{t})\right\}(\mathrm{p})=p \mathrm{~L}_{\alpha}\{\mathrm{f}(\mathrm{t})\}(\mathrm{p})-\mathbf{f}(0) .
$$

Proof. The proof is directly obtained by letting $n=0$ in Theorem 2.2.

Lemma 2.4 Let $\alpha \in\left(\frac{k-1}{k} 1\right], k \in N$ and $u(x, t)$ be $k \alpha$-differentiable real valued function. Then,

$$
\mathrm{L}_{\alpha}\left\{\frac{\partial^{\mathrm{k} \alpha}}{\partial \mathrm{t}^{\mathrm{k} \alpha}} \mathrm{u}(\mathrm{x}, \mathrm{t})\right\}(\mathrm{p})=\mathrm{p}^{\mathrm{k}} \mathrm{L}_{\alpha}\{\mathrm{u}(\mathrm{x}, \mathrm{t})\}(\mathrm{p})-\sum_{\mathrm{m}=0}^{\mathrm{k}-1} \mathrm{p}^{\mathrm{k}-\mathrm{m}-1} \frac{\partial^{\mathrm{m} \alpha}}{\partial \mathrm{t}^{\mathrm{m} \alpha}} \mathrm{u}(x, 0) .
$$

Proof. We can prove this theorem by mathematical induction on $k$.

For $k=1$, we have

$$
\mathrm{L}_{\alpha}\left\{\frac{\partial^{\alpha}}{\partial \mathrm{t}^{\alpha}} \mathrm{u}(x, \mathrm{t})\right\}(\mathrm{p})=p \mathrm{~L}_{\alpha}\{\mathrm{u}(x, \mathrm{t})\}(\mathrm{p})-\mathrm{u}(\mathrm{x}, 0),
$$

which is true by Corollary 2.3 .

Now, assume that the theorem is true for a particular value of $k$, say $r$. Then, we have

$$
\mathrm{L}_{\alpha}\left\{\frac{\partial^{r \alpha}}{\partial t^{r \alpha}} \mathrm{u}(x, t)\right\}(p)=p^{r} L_{\alpha}\{u(x, t)\}(p)-\sum_{m=0}^{r-1} p^{r-m-1} \frac{\partial^{m \alpha}}{\partial t^{m \alpha}} u(x, 0) .
$$

Now, we need to prove that the Theorem is true for $r+1$, that is

$$
\mathrm{L}_{\alpha}\left\{\frac{\partial^{(r+1) \alpha}}{\partial t^{(r+1) \alpha}} \mathrm{u}(x, t)\right\}(p)=p^{r+1} L_{\alpha}\{u(x, t)\}(p)-\sum_{m=0}^{r} p^{r-m} \frac{\partial^{m \alpha}}{\partial t^{m \alpha}} u(x, 0) .
$$

By using Theorem 2.4 and the assumption, we have

$$
\mathrm{L}_{\alpha}\left\{\frac{\partial^{(\mathrm{r}+1) \alpha}}{\partial \mathrm{t}^{(\mathrm{r}+1) \alpha}} \mathrm{u}(x, \mathrm{t})\right\}(\mathrm{p})=\mathrm{L}_{\alpha}\left\{\frac{\partial^{\alpha}}{\partial t^{\alpha}}\left(\frac{\partial^{r \alpha}}{\partial \mathrm{t}^{\mathrm{r} \alpha}} \mathrm{u}(x, t)\right)\right\}(p)
$$




$$
\begin{gathered}
=p L_{\alpha}\left\{\frac{\partial^{r \alpha}}{\partial t^{r \alpha}} u(x, t)\right\}(p)-\frac{\partial^{r \alpha}}{\partial t^{r \alpha}} u(x, 0) \\
=p\left[p^{r} L_{\alpha}\{u(x, t)\}(p)-\sum_{m=0}^{r-1} p^{r-m-1} \frac{\partial^{m \alpha}}{\partial t^{m \alpha}} u(x, 0)\right]-\frac{\partial^{r \alpha}}{\partial t^{r \alpha}} \mathfrak{u}(x, 0) \\
=p^{r+1} L_{\alpha}\{u(x, t)\}(p)-\sum_{m=0}^{r-1} p^{r-m} \frac{\partial^{m \alpha}}{\partial t^{m \alpha}} u(x, 0)-\frac{\partial^{r \alpha}}{\partial t^{r \alpha}} u(x, 0) \\
=p^{r+1} L_{\alpha}\{u(x, t)\}(p)-\sum_{m=0}^{r} p^{r-m} \frac{\partial^{m \alpha}}{\partial t^{m \alpha}} u(x, 0) .
\end{gathered}
$$

Therefore the theorem is true for every positive integral value of $k$.

In the following Lemma, we present the relation between the Katugampola Laplace transform and usual Laplace transform.

Lemma 2.5 Let $f:(0, \infty) \rightarrow R$ be a function such that $\alpha \in(n, n+1]$ and $L_{\alpha}\{f(t), p\}=\widetilde{f}(p)$. Then

$$
\mathrm{L}_{\alpha}\{f(t), p\}=\mathrm{L}\left\{f\left(((\alpha-n) t)^{\frac{1}{\alpha-n}}\right)\right\}(p)
$$

where,

$$
\mathrm{L}\{\boldsymbol{f}(x)\}(p)=\ell\{f(x)\}(p)=\int_{0}^{\infty} f(x) e^{-p x} d x
$$

denotes the usual Laplace transform.

Proof. By setting $y=\frac{t^{\alpha-n}}{\alpha-n}, t=((\alpha-n) y)^{\frac{1}{\alpha-n}}$ and $d y=t^{\alpha-n-1} d x$ in the formula

$$
\mathrm{L}_{\alpha}\{\mathrm{f}(\mathrm{t})\}(\mathrm{p})=\int_{0}^{\infty} \mathrm{f}(\mathrm{t}) \mathrm{e}^{-\mathrm{p} \frac{\mathrm{t}-\mathrm{n}-\mathrm{n}}{\alpha-\mathrm{n}}} \mathrm{t}^{\alpha-\mathrm{n}-1} \mathrm{dt},
$$

then, we have

$$
\begin{gathered}
L_{\alpha}\{f(t)\}(p)=\int_{0}^{\infty} e^{-p y} f\left(((\alpha-n) y)^{\frac{1}{\alpha-n}}\right) d y \\
=\int_{0}^{\infty} e^{-p t} f\left(((\alpha-n) t)^{\frac{1}{\alpha-n}}\right) d t=\ell\left\{f\left((\alpha-n) t^{\frac{1}{\alpha-n}}\right)\right\}(p) .
\end{gathered}
$$

Let us now present the Katugampola Laplace transform for some selected functions.

Theorem 2.6 Let $\alpha \in(n, n+1], n \in N$. We have the following transformations

$$
\begin{aligned}
& \text { i) } L_{\alpha}\left\{e^{ \pm \frac{t^{\alpha}-n}{\alpha-n} k^{2}}\right\}(p)=\ell\left\{e^{ \pm t k^{2}}\right\}(p)=\frac{1}{p \mp k^{2}} . \\
& \text { ii) } L_{\alpha}\left\{\sin \left(k \frac{t^{\alpha-n}}{\alpha-n}\right)\right\}(p)=\ell\{\sin (k t)\}(p)=\frac{k}{p^{2}+k^{2}} . \\
& \text { iii) } L_{\alpha}\left\{\cos \left(k \frac{t^{\alpha-n}}{\alpha-n}\right)\right\}(p)=\ell\{\cos (k t)\}(p)=\frac{p}{p^{2}+k^{2}} . \\
& \text { iv) } L_{\alpha}\left\{J_{0}\left(\frac{t^{\alpha-n}}{\alpha-n}\right)\right\}(p)=\ell\left\{J_{0}(t)\right\}(p)=\frac{1}{\sqrt{p^{2}+1}},
\end{aligned}
$$

where Bessel function [2] of order $n$ denoted by $J_{n}(t)$ is defined by $J_{n}(t)=\sum_{r=0}^{\infty} \frac{(-1)^{r}}{r ! ! \Gamma(n+r+1)}\left(\frac{t}{2}\right)^{n+2 r}, J_{0}\left(b \frac{t^{\alpha-n}}{\alpha-n}\right)$ is Bessel's function of order zero, 
the Laplace transform of $J_{0}(t)[10, p p .33-34]$ is $\frac{1}{\sqrt{1+p^{2}}}$, and the Laplace transform of $J_{1}(t)[10, p .35]$ is $1-\frac{p}{\sqrt{1+p^{2}}}$.

$$
\text { v) } \mathrm{L}_{\alpha}\left\{\mathrm{J}_{1}\left(\frac{\mathrm{t}^{\alpha-n}}{\alpha-\mathrm{n}}\right)\right\}(\mathrm{p})=\ell\left\{\mathrm{J}_{1}(\mathrm{t})\right\}(\mathrm{p})=1-\frac{\mathrm{p}}{\sqrt{\mathrm{p}^{2}+1}}
$$

where $J_{1}\left(\frac{t^{\alpha-n}}{\alpha-n}\right)$ is Bessel's function of order one.

$$
\text { vi) } \mathrm{L}_{\alpha}\left\{\operatorname{erf}\left(\sqrt{\frac{t^{\alpha-n}}{\alpha-n}}\right)\right\}(p)=\ell\{\operatorname{erf}(\sqrt{t})\}(p)=\frac{1}{p \sqrt{p+1}},
$$

where $\operatorname{erf}(\sqrt{\mathrm{t}})=\frac{2}{\sqrt{\pi}} \int_{0}^{\sqrt{t}} e^{-\mathfrak{u}^{2}} \mathrm{du}$ is an Error function.

$$
\text { vii) } \mathrm{L}_{\alpha}\left\{\mathrm{S}_{\mathrm{i}}\left(\frac{\mathrm{t}^{\alpha-n}}{\alpha-n}\right)\right\}(p)=\ell\left\{S_{i}(\mathrm{t})\right\}(\mathrm{p})=\frac{1}{\mathrm{p}} \tan ^{-1}\left(\frac{1}{\mathrm{p}}\right),
$$

where $S_{i}(t)=\int_{0}^{t} \frac{\sin u}{u} d u$, is a sine integral function.

$$
\text { viii) } L_{\alpha}\left\{C_{i}\left(\frac{t^{\alpha-n}}{\alpha-n}\right)\right\}(p)=\ell\left\{C_{i}(t)\right\}(p)=\frac{1}{2 p} \log \left(p^{2}+1\right),
$$

where $C_{i}(t)=\int_{0}^{t} \frac{\cos u}{u} d u$ is a cosine integral function.

$$
\text { x) } \mathrm{L}_{\alpha}\left\{\mathrm{E}_{\mathrm{i}}\left(\frac{\mathrm{t}^{\alpha-n}}{\alpha-\mathrm{n}}\right)\right\}(\mathrm{p})=\ell\left\{\mathrm{E}_{\mathrm{i}}(\mathrm{t})\right\}(\mathrm{p})=\frac{1}{\mathrm{p}} \log (\mathrm{p}+1),
$$

where $E_{i}(t)$ is a Exponential integral function.

$$
\text { xi) } \mathrm{L}_{\alpha}\left\{\mathrm{L}_{\mathrm{n}}\left(\frac{\mathrm{t}^{\alpha-n}}{\alpha-\mathrm{n}}\right)\right\}(\mathrm{p})=\ell\left\{\mathrm{L}_{\mathrm{n}}(\mathrm{t})\right\}(\mathrm{p})=\frac{(\mathrm{p}-1)^{\mathrm{n}}}{\mathrm{p}^{\mathrm{n}+1}},
$$

where $L_{n}(t)=\frac{e^{t}}{n !} \frac{d^{n}}{d t^{n}}\left(t^{n} e^{t}\right) d u$ is a Laguerre polynomial.

$$
\text { xii) } \mathrm{L}_{\alpha}\left\{\delta\left(\frac{\mathrm{t}^{\alpha-n}}{\alpha-\mathrm{n}}\right), \mathrm{k}\right\}=\ell\{\delta(\mathrm{t}), \mathrm{k}\}=1,
$$

where $\delta(t)$ is a Delta function.

Proof. We give the proofs of some transformations, where the rest of the proofs follows by using Definition 2.1, the substitution $y=\frac{t^{\alpha-n}}{\alpha-n}$, and then integration.

$$
\text { i) } \begin{aligned}
\mathrm{L}_{\alpha}\left\{e^{ \pm k^{2} \frac{t^{\alpha-n}}{\alpha-n}}\right\}(p)=\int_{0}^{\infty} e^{ \pm k^{2} \frac{t^{\alpha-n}}{\alpha-n}} e^{-p \frac{t^{\alpha-n}}{\alpha-n}} t^{\alpha-n-1} d t \\
=\int_{0}^{\infty} e^{\left(-p \pm k^{2}\right) \frac{t^{\alpha-n}}{\alpha-n} t^{\alpha-n-1} d t} \\
=\int_{0}^{\infty} e^{\left(-p \pm k^{2}\right) y} d y \\
=\ell\left\{e^{ \pm k^{2} y}\right\}(p) \\
=\frac{1}{p \mp k^{2}}, \text { where } y=\frac{t^{\alpha-n}}{\alpha-n} .
\end{aligned}
$$

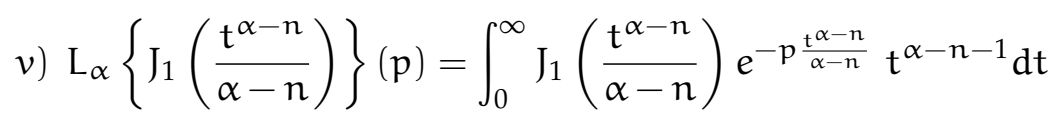

$$
=\int_{0}^{\infty} J_{1}(y) e^{-p y} d y=\ell\left\{J_{1}(y)\right\}(p)=1-\frac{p}{\sqrt{p^{2}+1}} .
$$




$$
\text { xii) } \begin{aligned}
\mathrm{L}_{\alpha}\left\{\delta\left(\frac{\mathrm{t}^{\alpha-n}}{\alpha-n}\right), \mathrm{k}\right\} & =\int_{0}^{\infty} \delta\left(\frac{\mathrm{t}^{\alpha-n}}{\alpha-n}\right) e^{-\mathrm{p}^{\frac{\mathrm{t}^{\alpha}-\mathrm{n}}{\alpha-n}}} \mathrm{t}^{\alpha-n-1} \mathrm{dt} \\
= & \int_{0}^{\infty} \delta(\mathrm{y}) \mathrm{e}^{-\mathrm{py}} \mathrm{dy} \\
& =\ell\{\delta(\mathrm{y}), \mathrm{k}\}=1,
\end{aligned}
$$

where the Laplace transform of the delta function is given by

$$
\ell\{\delta(t-a) ; p\}=\int_{0}^{+\infty} \delta(t-a) e^{-p t} d t=e^{-a p}
$$

and when $a=0$, we obtain the result $\ell\{\delta(t) ; p\}=\int_{0}^{+\infty} \delta(t) e^{-p t} d t=1$.

Theorem 2.7 Let $\alpha \in(n, n+1]$ and $\mathrm{L}_{\alpha}\{f(t), p\}=\widetilde{f}(p), L_{\alpha}\{g(x), p\}=\widetilde{g}(p)$. Then

$$
\text { i) } \mathrm{L}_{\alpha}\left\{\mathrm{t}^{\mathrm{m}}\right\}(\mathrm{p})=\frac{(\alpha-\mathrm{n})^{\frac{\mathrm{m}}{\alpha-n}}}{\mathrm{p}^{1+\frac{m}{\alpha-n}}} \Gamma\left(1+\frac{\mathrm{m}}{\alpha-n}\right), \quad \mathrm{p}>0 .
$$

where $\Gamma(t)$ is the Gamma function.

$$
\text { ii) } \mathrm{L}_{\alpha}\{\mathrm{H}(\mathrm{t}-\mathrm{a})\}(\mathrm{p})=\frac{\mathrm{e}^{-\left(\frac{\mathrm{a}^{\alpha-n}}{\alpha-n}\right) p}}{\mathrm{p}} .
$$

where $\mathrm{H}(\mathrm{t}-\mathrm{a})$ is the Unit Step (or Heaviside's unit) function.

Proof. We can prove $i$ ) by setting $t^{m}=\left[\left(\frac{\alpha-n}{p}\right) u\right]^{\frac{m}{\alpha-n}}$.

In particular,

A) if $m=0$ then, $\mathrm{L}_{\alpha}\{1\}(\mathrm{p})=\frac{1}{\mathrm{p}} \Gamma(1)=\frac{1}{\mathrm{p}}$,

B) if $m=1$ then, $L_{\alpha}\{t\}(p)=\frac{(\alpha-n)^{\frac{1}{\alpha-n}}}{p^{1+\frac{1}{\alpha-n}}} \Gamma\left(1+\frac{1}{\alpha-n}\right)$.

But for the result $i i$ ), we can prove it by setting $u=\frac{t^{\alpha-n}}{\alpha-n}$.

We next turn to obtain some important properties of the Katugampola Laplace transform.

Lemma 2.8 Katugampola Laplace transform $\mathrm{L}_{\alpha}\{f(x), \mathrm{K}\}$ is liner. That is

$$
\mathrm{L}_{\alpha}\{a f+b g\}=a L_{\alpha}\{f\}+b L_{\alpha}\{g\}
$$

The proof is trivial.

\section{Theorem 2.9 (The Shifting Property)}

By introducing the Katugampola Laplace transform

$$
L_{\alpha}\left\{e^{-a \frac{t^{\alpha-n}}{\alpha-n}} f(t)\right\}(p)=\widetilde{f}(p+a)=L\left\{e^{-a \cdot t} f\left(((\alpha-n) t)^{\frac{1}{\alpha-n}}\right)\right\}(p),
$$

where $\tilde{f}(p)=L_{\alpha}\{f(t)\}(p)$, the following results are readily follow:

$$
\begin{gathered}
\text { (i) } \mathrm{L}_{\alpha}\left\{e^{-a \frac{t^{\alpha-n}}{\alpha-n}} t^{m}\right\}(p)=\frac{(\alpha-n)^{\frac{m}{\alpha-n}}}{(p+a)^{\frac{m}{\alpha-n}+1}} \Gamma\left(1+\frac{m}{\alpha-n}\right) . \\
\text { (ii) } L_{\alpha}\left\{e^{-a \frac{t^{\alpha-n}}{\alpha-n}} e^{-b \frac{t^{\alpha-n}}{\alpha-n}}\right\}(p)=\frac{1}{(p+a)-b} .
\end{gathered}
$$




$$
\begin{aligned}
& \text { (iii) } L_{\alpha}\left\{e^{-a \frac{t^{\alpha-n}}{\alpha-n}} \sin \left(b \frac{t^{\alpha-n}}{\alpha-n}\right)\right\}(p)=L\left\{e^{-a t} \sin (t)\right\}(p)=\frac{b}{(p+a)^{2}+b^{2}} . \\
& \text { (iv) } L_{\alpha}\left\{e^{-a \frac{t^{\alpha-n}}{\alpha-n}} \cos \left(b \frac{t^{\alpha-n}}{\alpha-n}\right)\right\}(p)=L\left\{e^{-a t} \cos (t)\right\}(p)=\frac{p+a}{(p+a)^{2}+b^{2}} .
\end{aligned}
$$

\section{Theorem 2.10 (Change of Scale Property)}

If $\mathrm{L}_{\alpha}\{f(t)\}(p)=\widetilde{f}(p)$ then,

$$
\mathrm{L}_{\alpha}\{\mathrm{f}(\mathrm{at})\}(\mathrm{p})=\frac{1}{\mathrm{a}^{\alpha-n}} \tilde{\mathrm{f}}\left(\frac{\mathrm{p}}{\mathrm{a}^{\alpha-n}}\right) .
$$

Proof. Starting with

$$
\mathrm{L}_{\alpha}\{\mathrm{f}(\mathrm{at})\}(\mathrm{p})=\int_{0}^{\infty} e^{-\mathrm{p} \frac{\mathrm{t}^{\alpha-n}}{\alpha-n}} f(a \mathrm{t}) \mathrm{t}^{\alpha-n-1} \mathrm{dt},
$$

putting $u=a t$, so that $t=\frac{u}{a}$ and $d u=a d t($ as $t: 0 \rightarrow \infty \Rightarrow u: 0 \rightarrow \infty)$,

we have

$$
\begin{gathered}
L_{\alpha}\{f(u)\}(p)=\int_{0}^{\infty} e^{-p \frac{1}{\alpha-n}\left(\frac{u}{a}\right)^{\alpha-n}} f(u)\left(\frac{u}{a}\right)^{\alpha-n-1} \frac{1}{a} d u \\
=\frac{1}{a^{\alpha-n}} \int_{0}^{\infty} e^{-\left(\frac{p}{a^{\alpha-n}}\right) \frac{u^{\alpha-n}}{\alpha-n}} f(u) u^{\alpha-n-1} d u=\frac{1}{a^{\alpha-n}} \tilde{f}\left(\frac{p}{a^{\alpha-n}}\right) .
\end{gathered}
$$

Theorem 2.11 Let $L_{\alpha}\{f(t)\}(p)=\widetilde{f}(p)$ then,

$$
L_{\alpha}\left\{\left(\frac{t^{\alpha-n}}{\alpha-n}\right)^{m} f(t)\right\}(p)=(-1)^{m} \frac{d^{m}}{d p^{m}} \tilde{f}(p),
$$

where $\mathrm{m}=1,2,3, \ldots$.

Proof. We can prove this Theorem by mathematical induction on $\mathrm{m}$.

Theorem 2.12 (Convolution Theorem) Let $g(t)$ and $h(t)$ be arbitrary functions. Then,

$$
\mathrm{L}_{\alpha}\{\mathrm{g} * \mathrm{~h}\}=\mathrm{L}_{\alpha}\{\mathrm{g}(\mathrm{t})\} \mathrm{L}_{\alpha}\{\mathrm{h}(\mathrm{t})\}=\mathrm{L}_{\alpha}\{\mathrm{g}\} \mathrm{L}_{\alpha}\{\mathrm{h}\},
$$

where $g * h$ is the Convolutions of function $g(t)$ and $h(t)$ defined as

$$
g * h=\int_{0}^{t} g(x) h(t-x) d x .
$$

Proof. It is easy to prove the results by using Lemma 2.5, and the definition of Laplace transform.

Remark 2.13 Let $g(x)$ and $h(x)$ be arbitrary functions, and let $L_{\alpha}{ }^{-1}\{\widetilde{g}(p)\}=g(x)$ and $L_{\alpha}{ }^{-1}\{\widetilde{h}(p)\}=$ $h(x)$. Then,

$$
(g * h)(t)=\mathrm{L}_{\alpha}{ }^{-1}\left\{\mathrm{~L}_{\alpha}\{(\mathrm{g} * \mathrm{~h})(\mathrm{t})\}\right\}=\mathrm{L}_{\alpha}{ }^{-1}\left\{\mathrm{~L}_{\alpha}\{\mathrm{g}(\mathrm{t})\} \mathrm{L}_{\alpha}\{\mathrm{h}(\mathrm{t})\}\right\} .
$$

\section{Conclusions}

In this paper, we obtained several results that have close resemblance to the results found in classical calculus. We defined a new kind of fractional Laplace transform. Also we gave some prosperities of this transform which is considered as a generalization to the usual Laplace transform. 


\section{References}

[1] T.Abdeljawad, On Conformable Fractional Calculus, J. Comput. Appl. Math., 279(2015) 57-66. https:/ / doi.org/10.1016/j.cam.2014.10.016

[2] B.G. Arfken, J. H.Weber and F. E.Harris, Mathematical Methods for Physicists, 7th ed. Orlando, FL: Academic Press, (2013).

[3] U.N. Katugampola, A new fractional derivative with classical properties, J.American Math. Soc., arXiv: 1410.6535v2 (2014).

[4] A.A. Kilbas, H.M.Srivastava and J.J. Trujillo, Theory and Applications of Fractional Differential Equations, Elsevier B.V., Amsterdam, Netherlands, 2006.

[5] R.Khalil , M. Al Horani, A. Yousef and M. Sababheh,A new definition of fractional derivative, Journal of Computational and Applied Mathematics, vol. 264, pp. 65-70, 2014.

[6] K.B. Oldham, J. Spanier, The fractional calculus, Academic Press, New York, 1974.

[7] I. Podlubny, "Fractional Differential Equations ", Academic Press, San Diego. California, USA, 1999.

[8] S.G. Samko, A.A. Kilbas and O.I. Marichev, Fractional Integrals and Derivatives : Theory and Applications, Gordon and Breach, Yverdon et alibi, 1993.

[9] T.O.Salim, A.A.K. Abu Hany, M.S.El-Khatib, On Katugampola Fourier Transform, Hindawi, Journal of Mathematics, vol. 2019, Article ID 5942139, 6 pages, 2019. 